\title{
Construction, identification, and immunogenic assessments of an HSV-1 mutant vaccine with a UL18 deletion
}

\author{
L. WANG ${ }^{1,2,3}$, Q. AOLI WANG ${ }^{1,2 \#}$, F. JIN ${ }^{1,2,3}$, S. FANG ${ }^{1,2,3}$, F. LUO $^{1,2,3}$, Y. WU ${ }^{1,2,3}$, F. LI ${ }^{1,2,4}$, J. LIU ${ }^{1,2,3}$, Y. WANG ${ }^{1,2,4}$, \\ J. JIN ${ }^{1,2}$, X. LIAO $^{1,2}$, Z. REN ${ }^{1,2^{*}}$, Y. WANG ${ }^{1,2^{*}}$
}

\begin{abstract}
${ }^{1}$ Guangzhou Jinan Biomedicine Research and Development Center, National Engineering Research Center of Genetic Medicine, Jinan University, Guangzhou, Guangdong, P. R. China; ${ }^{2}$ Key Laboratory of Bioengineering Medicine, Guangzhou, Guangdong, P. R. China; ${ }^{3}$ College of LifeScience and Technology, Jinan University, Guangzhou, Guangdong, P. R. China; ${ }^{4}$ College of Pharmacy, Jinan University, Guangzhou, Guangdong, P. R. China
\end{abstract}

\begin{abstract}
Summary. - Herpes simplex virus type 1 (HSV-1) is a mucosal and nerve pathogen, whose morbidity shows an increasing tendency. Although several antiviral drugs exist, there is no cure for viral latency for virtually all carriers. There is an urgent need for an HSV-1 vaccine to control infection and limit its spread and recurrence. The UL18 gene, encoding a vital component of capsids, is one of the essential genes of HSV-1. Deletion of UL18 from HSV-1 may be exploited as a new approach to develop an attenuated vaccine. The purpose of this study was to construct a DNA vaccine with a full-length UL18 gene deletion of the HSV-1 genome that can induce an effective immune response. A UL18-knockdown plasmid (BAC-HSV-1 $\Delta$ UL18) was constructed using the bacterial markerless gene knockout system, consisting of the functional pREDI plasmid and BAC-HSV-1 plasmid. Mice were immunized weekly for 3 weeks, and at 1 week post immunization, blood and splenocyte samples of vaccinated and control groups of mice were prepared for immunogenicity assessment. The level of immune response was evaluated using a DTH assay, cytokine determination, and splenocyte proliferation assay. Combination of the pREDI plasmid and BAC-HSV-1 plasmid provides an effective bacterial markerless gene knockout system. Using two-step homologous recombination with the UL18 homologous recombination fragment constructed by multistep PCR amplification, BAC-HSV-1 $\Delta$ UL18 plasmid vaccine was successfully constructed and was found to significantly enhance cellular immune responses.
\end{abstract}

Keywords: homologous recombination; UL18 gene; HSV-1; gene knockout; immunogenicity

\section{Introduction}

As an extremely common pathogen worldwide, HSV-1 causes infections primarily of the lip, pharynx, eye, central nervous system, and, occasionally, genitals. Moreover,

*Corresponding author. E-mail: rz62@163.com, twang-yf@163. com; phone: $+86-020$ 85220504. ${ }^{*}$ Authors contributed equally to this work.

Abbreviations: $\mathrm{BAC}=$ bacterial artificial chromosome; $\mathrm{BAC}-$ HSV-1 = the HSV-1 strain 17 genome inserted into the UL37/ UL38 inter genic region; BAC-HSV-1 $\triangle \mathrm{UL} 18=$ full-length UL18 gene deletion of the HSV-1 genome plasmid; DTH = delayed type hypersensitivity; HSV-1 = herpes simplex virus type 1
HSV-1 has a certain relationship with Alzheimer's disease (Piacentini et al., 2014), sporadic encephalitis (Kennedy and Chaudhuri, 2002), AIDS-related complications (Weller, 2011), multiple tumors (Jensen et al., 2010) and disseminated disease (Boivin et al., 2006). HSV is persistent as it establishes a life-long latent infection within the nervous system and eliminates the host immune response. Thus, developing an efficient HSV vaccine is an ideal way to control its infection and limit its spread and recurrence.

HSV-1 is a spherical virus consisting of an envelope, tegument, capsid, and genomic DNA (Grunewald and Cyrklaff et al., 2006). Among the eleven glycoproteins located on the HSV-1 envelope, gD and gB have been shown to be important HSV-1 immunogens, inducing cellular and humoral immunity 
(Jin et al., 2001). The capsid, one of the most crucial structural components for protecting DNA, is an icosahedron composed of 11 pentamer molecules and 150 hexamer molecules of capsid protein VP5. VP23, encoded by the UL18 gene, is an essential capsid protein, and a key component of the triplex structure that links the pentamers and hexamers. The triplex, which contains two units of VP23 and a single unit of VP19C, is important for stabilizing the capsid shell structure (Trus et al., 1996). Interference of protein VP23 greatly affects virus packaging and replication (Jin et al., 2014). In short, HSV-1 with mutant UL18 has the potential to be used to create a DNA vaccine to stimulate immune responses in vivo.

DNA vaccines are developed using molecular biology techniques, which make use of the cell system of a host to express an antigen gene from a naked DNA plasmid. As the third generation of vaccines after subunit and attenuated live virus vaccines, DNA vaccines offer immense advantages compared with previous vaccines. DNA vaccines complete antigen synthesis and presentation in vivo, which induces immune responses of $\mathrm{CD}^{+} \mathrm{T}$ cells through MHC-I recognition (Rodrigue et al., 1997). The process, by which DNA vaccines invoke immunogenicity, is similar to that of natural viral infection as opposed to that of subunit and attenuated live virus vaccines.

Here, we present the bacterial markerless gene knockout system to produce a UL18 knockout HSV-1 plasmid (BACHSV-1 $\triangle$ UL18) by using two-step homologous recombination. We further found that BAC-HSV-1 $\Delta$ UL18 induced a great cellular immune effect in mice. This suggests the potential significance of utilizing the UL18 knockout HSV-1 DNA vaccine, which can be combined with an attenuated live virus vaccine by using the markerless gene knockout system in vitro.

\section{Materials and Methods}

Cells, bacteria, and plasmids. African green monkey kidney cells (Vero) (ATCC CCLS1), purchased from American Type Culture Collection, were maintained in Dulbecco's Modified Eagle Medium (Gibco, USA) supplemented with $10 \%$ fetal bovine serum (FBS) at $5 \% \mathrm{CO}_{2}$ in a humidified $37^{\circ} \mathrm{C}$ incubator. Vero cells were used in virus rescue and antibody neutralization assays. Escherichia coli K-12 strain MG1655 was used in all markerless gene knockdown experiments. E. coli stain $\mathrm{DH} 5$ a, as a cloning host, was used for plasmid transformation and extraction. All strains were grown in Luria Broth (LB). Plasmids pKD46, BAC, and BAC-HSV-1 are stored in our laboratory. Plasmid pKD46, with $\mathrm{Cm}^{\mathrm{r}}$ and $\mathrm{Amp}^{\mathrm{r}}$ resistance, carries an arabinose-inducible promoter driving $\lambda$-Red recombinase expression. Plasmid BAC-HSV-1, acquired from Gierasch et al. (2006) has the BAC sequence inserted into the $U_{\mathrm{L}} 37 / U_{\mathrm{L}} 38$ intergenic region of HSV-1 strain 17 genome. The bacterial artificial chromosome (BAC) sequence is flanked by cre/LoxP sites with $\mathrm{Cm}^{\mathrm{r}}$ resistance, with loxP site-flanked BAC sequences removed by cre recombinase in vitro. Then the mixture of BAC and HSV-1 sequences was separated by DNA electrophoresis. Plasmids pREDI $\left(\mathrm{Amp}^{\mathrm{r}}\right)$ and BAC-HSV-1 $\triangle \mathrm{UL} 18\left(\mathrm{Cm}^{\mathrm{r}}\right)$ were constructed in our laboratory. Plasmid pREDI was constructed from plasmid pKD46 by insertion of I-SecI-encoded gene under the control of rhamnose promoter PrhaB using the $\mathrm{NcoI}$ restriction site. The construction strategy of plasmid BAC-HSV-1 $1 \Delta$ U18 is illustrated in the results. The plasmid inducible conditions used were $\mathrm{LB}$ with a final concentration of $10 \mathrm{mM}$ arabinose or rhamnose. The lethal concentration of sucrose for the SacB induction site on pREDI is $5 \%$ of the LB.

PCR amplification. PCR was performed in a $50 \mu \mathrm{l}$ reaction mixture containing $25 \mu \mathrm{l}$ of $2 \times$ PrimeSTAR $^{\oplus}$ GC buffer, $4 \mu \mathrm{l}$ of $2.5 \mathrm{mmol} / \mathrm{l} \mathrm{dNTP}$ mixture, $1.5 \mu \mathrm{l}$ each of $20 \mu \mathrm{mol} / \mathrm{l}$ forward and reverse primers, $<200 \mathrm{ng}$ of template DNA, $0.5 \mu$ l of PrimeSTAR ${ }^{\infty}$ HS DNA Polymerase $(2.5 \mathrm{U} / \mu \mathrm{l}$, Takara), and up to $50 \mu \mathrm{l}$ of triple distilled water in a tube (Eppendorf). Reaction conditions consisted of an initial denaturation step at $94^{\circ} \mathrm{C}$ for $3 \mathrm{~min}$, followed by 30 cycles of $98^{\circ} \mathrm{C}$ for $1 \mathrm{~s}, 60^{\circ} \mathrm{C}$ for $5 \mathrm{~s}$ and $72^{\circ} \mathrm{C}$ for $1 \mathrm{~min} / \mathrm{kb}$, with a final extension at $72^{\circ} \mathrm{C}$ for $5 \mathrm{~min}$. The PCR products were analyzed by $1 \%$ agarose gel electrophoresis. The E.Z.N.A gel extraction Kit (Omega Bio-Tek) was utilized for gel extraction. The PCR primer sequences used are as follows: I-SceI: 5'-TTA GAC TGG TCG TAA TGA AAT TCA GCA GGA TCA CAT CTG GGT C-3' and 5'-CAT GCC ATG GGT CGA CTT ATT TCA GGA AAG TTT CGG AGG AGA TAG TG-3'; A-C: 5'-CCC ACC CCC CCG TGG GTC TAG CCG GGC CGG CGC CGA TCCACG CGG CAG G-3'; Kan²: 5'-TGG CTT TGT TGA ATA AAT CGC GAC CAC GGC CAGAGC GACCCG TCC-3' and 5'-GGA CGG GTC GCT CTG GCC GTG GTC GCG ATT TAT TCA ACA AAG CCA CG-3'; SacB: 5'-CTG GCA ATT ACC CTG TTA TCC CTA GGC CCG TAG TCT GCAAAT CCT TTT-3' and 5'-CAT CGC GAT ACC CTC GGG CAT CTC GCA TCT TGC AAG AATGGG CCT CGT T-3'; A-C-Kan ${ }^{\mathrm{r}}-\mathrm{SacB}-\mathrm{B}$ : 5'TGG ATG CCC ACC CCC ACC CCC CCG TGG GTC TAG CCG GGC-3' and 5'-ATG CTG GCG GAC GGC TTT GAA ACT GAC ATC GCG ATA CCC TCG GGC ATC TCG-3'.

Electroporation and purification of plasmids. Electrocompetent recipient cells were prepared from E. coli strain DH5a with BACHSV-1 and pREDI plasmids. The preparation method was that the E. coli strain DH5a with BAC-HSV-1 and pREDI plasmids were washed and resuspended in $10 \%$ of the glycerinum solution three times in low temperature conditions. Electroporation shock conditions were $1500 \mathrm{~V}$ for $40 \mu$ s (Eppendorf). Plasmids were purified using PureLink ${ }^{\circledR}$ HiPure Plasmid Filter DNA Purification Kits (Invitrogen, USA), and stored in sterilized triple-distilled water. The total yields of BAC-HSV-1 $\triangle \mathrm{UL} 18, \mathrm{BAC}$ and BAC-HSV-1 plasmids were $4.2 \mathrm{mg}$, $4.2 \mathrm{mg}$ and $1.8 \mathrm{mg}$, respectively. Meanwhile, the A260/ A280 values were greater than 1.8 .

Transfection. Vero cells were seeded into 6-well cell culture plates and grown to $70 \%$ confluence for plasmid transfection. Plasmids were dissolved in Opti-MEM, siRNA-Mate was subsequently added, and the transfection complex was added directly to Vero cells. Cells were observed for the cytopathic effect.

Immunofluorescence. After transfection, Vero cells were stained with VP5 antibody (Abcam) and DAPI (Beyotime Biotechnology). 
Fluorescence images were acquired using an OLYMPUS fluorescence microscope (OLYMPUS IX71).

Mouse immunity. Bilateral musculus biceps brachii injections were conducted in male Kunming mice (Laboratory Animals Monitoring Institute, Guangdong) at 5-weeks of age, with $100 \mu \mathrm{g}$ per mouse administered once a week for 3 weeks. There were 4 groups, namely the negative control, positive control, experimental group, and normal control, which received BAC, BAC-HSV-1, BAC-HSV-1 $\triangle$ UL18, and PBS, respectively. One week post the third triplicate-vaccination, blood was collected from the orbits and splenic lymphocytes were extracted in each group.

Sample preparation. One week post final immunization, mice were euthanized by cervical dislocation. To prepare blood samples, eyeballs were extirpated to draw blood, and the blood collected by heparin sodium-anticoagulation tube was centrifuged at $4^{\circ} \mathrm{C}, 2,500 \mathrm{x}$ g for $5 \mathrm{~min}$. The supernatants were extracted for IgG determination and antibody neutralization assays. To collect splenocyte samples, spleens were excised, cut into pieces in DMEM, and digested by adding pancreatin for $30 \mathrm{~min}$. Subsequently, the splenocytes were collected using an $80-\mathrm{mm}$ mesh, centrifuged at $150 \mathrm{x} \mathrm{g}$ for $5 \mathrm{~min}$ and allowed to rest for $5 \mathrm{~min}$. After addition of $\mathrm{NaCl}(3.6 \% ; 1 \mathrm{ml})$, the splenocytes were washed with DMEM twice. These splenocytes were used for the detection of cytokines and in the cell proliferation assays.

Delayed type hypersensitivity assay. One week after the last immunization, the right subcutaneous auricle of vaccinated mice was injected with inactivated HSV-1 suspension $(10 \mu \mathrm{l})$, and the left was injected with supernatant of the frozen lysate $(10 \mu \mathrm{l})$. The thickness of auricles was measured $48 \mathrm{~h}$ later using a vernier caliper. Data are represented as DTH, which is calculated by subtracting the measurement for the left subcutaneous auricle from the right one.

Determination of cytokines by ELISA. Splenocytes were diluted to a final concentration of $5 \times 10^{6}$ cells $/ \mathrm{ml}$ in DMEM supplemented with $10 \%$ FCS in 24-well plates. Appropriately diluted plasmid solutions were added to the wells $(10 \mu \mathrm{g} /$ well), as well as normal and negative controls. After $48 \mathrm{~h}$, the supernatants of the wells were collected and the levels of cytokines (IL-2, IL-4, L-10, and IFN $-\gamma$ ) in the supernatants of splenocytes from the vaccinated and control groups were measured using the Cytokine ELISA kit, as per manufacturer's instructions.

Determination of IgG by ELISA. IgG was determined in immune mice sera by ELISA method. Briefly, the wells of the flat bottom plates were coated with the inactivated HSV-1 liquid (100 $\mu \mathrm{l} /$ well). $3 \%$ of BSA (Fraction V) were added to the wells for $2 \mathrm{~h}$ at room temperature, so that the wells are covered. After washing, the plate was probed with appropriately vaccinated sera and pre-immune sera as negative control. This was followed by $1 \mathrm{~h}$ incubation with 1:1000 dilution of HRP mutton anti-mouse-IgG conjugate at room temperature, several washes and $100 \mu \mathrm{l}$ of $\mathrm{O}$-phenylenediamine was added. The reaction was stopped after $10 \mathrm{~min}$ by adding $0.2 \mathrm{~mol} / \mathrm{l}$ of $\mathrm{H}_{2} \mathrm{SO}_{4}$ and the $\mathrm{OD}$ was read at $492 \mathrm{~nm}$.

Cell proliferation assay. Proliferation of splenocytes from the immunized and control groups of mice was measured by the MTT cell proliferation assay. Briefly, the splenocytes were diluted to a final concentration of $5 \times 10^{6} \mathrm{cells} / \mathrm{ml}$ in DMEM supplemented with $10 \%$ FCS in 96-well plates. After $24 \mathrm{~h}$, cells were treated with plasmids $(10 \mu \mathrm{g} / \mathrm{ml})$. After $72 \mathrm{~h}$, the proliferation of the splenocytes was measured by adding MTT. Data are represented as stimulation index (SI), which is calculated by dividing the absorbance (OD) observed in experimental group by the OD observed in negative cells.

Neutralization antibody assay. The mixtures of HSV-1 virus (100 TCID $_{50}$ ) and BAC- $\Delta$ UL18-, BAC-HSV-1- or BAC-vaccinated sera, respectively, by double dilution were added to DMEM supplemented with $2 \%$ inactivated FCS. The Vero cells were inoculated in 96-well plates ( $100 \mu \mathrm{l} /$ well, 3 repeated wells of one concentration). The plates were subsequently incubated at $37^{\circ} \mathrm{C}$ for 5 days. Then the degree of cytopathic effect was recorded by observing the Vero cells in an optical microscope (LEICA). The symbol "+" and "-" represents the degree of cytopathic effect. “+”, 1\% 25\% of Vero cells in one well have cytopathic effect; "++", $25 \%$ 50\% of Vero cells in one well have cytopathic effect; "+++", 50\% 75\% of Vero cells in one well have cytopathic effect; “++++", 75\% 100\% of Vero cells in one well have cytopathic effect; “-”, none of Vero cells in one well has cytopathic effect.

Statistical analysis. Statistical analysis was performed using Prism 5 software (GraphPad). Two-tailed Student's t-test was used to determine the significance of difference between two groups. ${ }^{*} \mathrm{P}<0.05 ;{ }^{* *} \mathrm{P}<0.01$. The data were presented as mean \pm standard error of the mean.

\section{Results}

Construction of the bacterial markerless gene knockout system

The pREDI plasmid is the core of the bacterial gene knockout system, which contains two independent in-

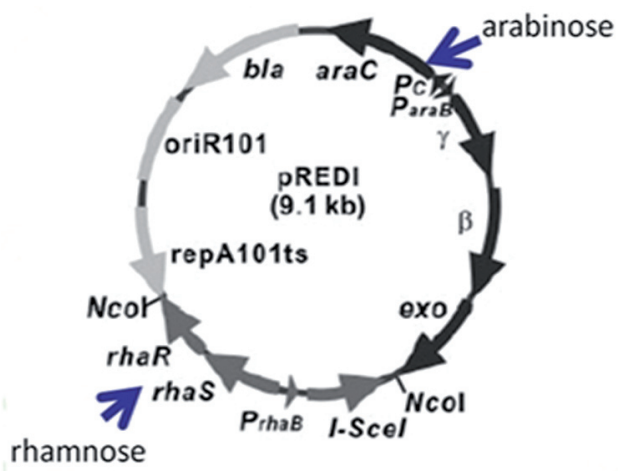

Fig. 1

pREDI plasmid profile

The pREDI plasmid, $9.1 \mathrm{~kb}$ in length, provides (i) arabinose-inducible (promoter $=$ ParaB) $\lambda$-Red recombinase functions in the replacement of a target genomic region with a linear DNA cassette and (ii) rhamnoseinducible (promoter $=$ PrhaB) I-SceI endonuclease functions in markerless deletion. 
ducible promoters to express two different enzymes (Yu et al., 2008): (i) $\lambda$-Red recombination protein expression driven by an arabinose-inducible promoter, and (ii) I-SceI endonuclease expression driven by a rhamnose-inducible promoter (Fig. 1), designed for two-step homologous recombination. As the functional plasmids, pREDI plasmids were transformed to E. coli with BAC-HSV-1 plasmids, constructing the bacterial markerless gene knockout system.

\section{Construction of UL18-deletion BAC-HSV-1 vaccine}

Through the above constructed in vitro viral gene knockout system, we deleted the VP23-coding gene by using two-step homologous recombination. Taking the target UL18 gene as a central point marked by the B gene, the sequence upstream of it was $\mathrm{A}$ and that downstream was C. To build the UL18 homologous recombination fragment with forward and reverse screening maker genes, we

(A)
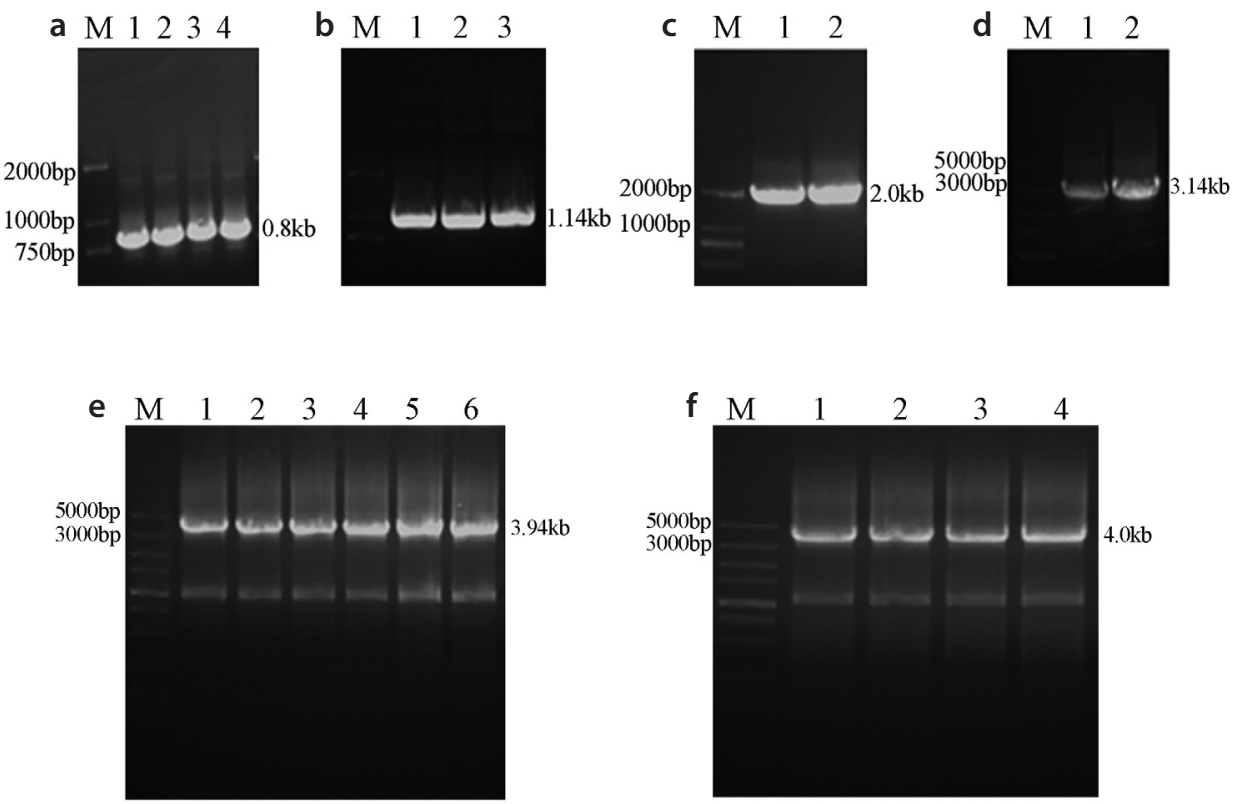

(B)

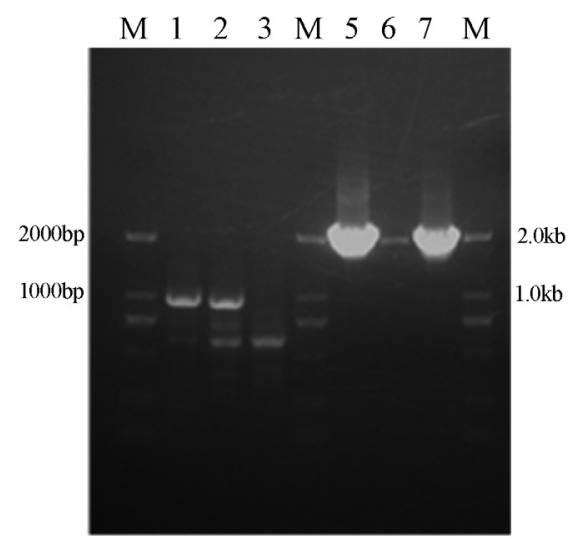

(C)

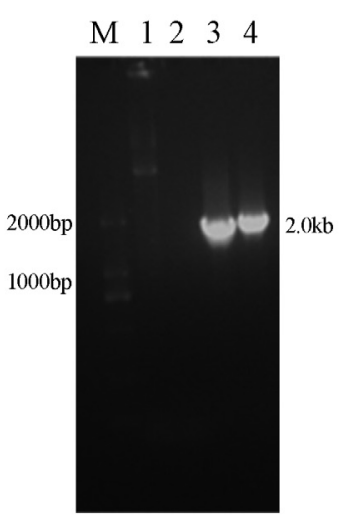

Fig. 2

Construction of the UL18-deletion BAC-HSV-1 plasmid

(A) The UL18 homologous recombination fragment (A-C-Kan ${ }^{\mathrm{r}}$-SacB-B) was constructed by PCR and analyzed by $1 \%$ agarose gel electrophoresis: (a) line M: DL 2000; line 1-4: segment C, product size $0.8 \mathrm{~kb}$; (b) line 1-3: segment Kan', product size $1.14 \mathrm{~kb}$; (c) line 1-2: SacB, product size $2.0 \mathrm{~kb}$; (d-f) line M: DL 5000; (d) line 1-2: segment Kann ${ }^{\mathrm{r}}-\mathrm{SacB}-\mathrm{B}$, product size $3.14 \mathrm{~kb}$; (e) line 1-6: C-Kan ${ }^{\mathrm{r}}$-SacB-B, product size $3.94 \mathrm{~kb}$; (f) line 1-4: full deletion cassette, product size $4.0 \mathrm{~kb}$. (B-C) The Kan ${ }^{\mathrm{r}}$ and SacB marking genes were removed by the homologous recombination induced by rhamnose: line M: DL 2000; (B) line 1-3: UL18, product size $1.0 \mathrm{~kb}$; line 5-7: SacB, product size $2.0 \mathrm{~kb}$; (C) line 1-4: SacB, product size $2.0 \mathrm{~kb}$. 
amplified the downstream target gene set by PCR first, with a product size of approximately $0.8 \mathrm{~kb}$, which was ligated to a roughly $50 \mathrm{bp}$ section upstream of the UL18 gene and called A-C fragment (Fig. 2Aa). Likewise, PCR amplification of kanamycin-resistant gene fragments as a forward screening maker gene $\left(\mathrm{Kan}^{\mathrm{r}}\right)$ (Fig. 2Ab) and the levansucrase-coding gene as a reverse screening marker (SacB-B) was performed, and they were ligated to a roughly $50 \mathrm{bp}$ terminal fragment of UL18 (Fig. 2Ac). Using overlapping PCR twice and ligating $\mathrm{Kan}^{\mathrm{r}}$ and $\mathrm{SacB}-\mathrm{B}\left(\mathrm{Kan}^{\mathrm{r}}-\mathrm{SacB}-\mathrm{B}\right)$ as well as $\mathrm{Kan}^{\mathrm{r}}-\mathrm{SacB}-\mathrm{B}$ (Fig. 2Ad-e) and A-C successively (Fig. 2Af), we ultimately obtained the UL18 homologous recombination fragment (A-C-Kan ${ }^{\mathrm{r}}-\mathrm{SacB}-\mathrm{B}$, product size approximately $4.0 \mathrm{~kb}$ ).

Next, using homologous recombination, the A-C-Kan ${ }^{\mathrm{r}}-$ SacB-B fragment was inserted into the correct site of BAC$\mathrm{HSV}-1 . \mathrm{A}-\mathrm{C}-\mathrm{Kan}^{\mathrm{r}}-\mathrm{SacB}-\mathrm{B}$ fragments were electroporated into electrocompetent recipient cells with BAC-HSV-1 and arabinose-inducible $\lambda$-Red recombinase expression. The electrocompetent recipient cells were incubated in SOC culture at $37^{\circ} \mathrm{C}$ for $1-2 \mathrm{~h}$. The forward screening maker gene, $\operatorname{Kan}^{\mathrm{r}}$, was used to screen for bacteria with successful recombination, where the UL18 gene was replaced by the marker genes. Then, we validated the monoclone by PCR amplification of the UL18 gene and two marker genes, which showed that the positive control DNA (BAC-HSV-1) amplified the UL18 gene, unlike the selected monoclone and negative control DNA (BAC) that could only amplify the Kan ${ }^{\mathrm{r}}$ and $\mathrm{SacB}$ gene products (Fig. $2 \mathrm{~B}-\mathrm{C}$ ). Thus, the Kan ${ }^{\mathrm{r}}-\mathrm{SacB}$ gene fragment had been recombined into the correct site to replace the $U L 18$ gene.

The second step of the homologous recombination was to remove the forward and reverse screening marker genes including $\operatorname{Kan}^{\mathrm{r}}$ and $\mathrm{SacB}$ to eliminate the potential negative influence of the marker genes on viral proliferation. First, rhamnose was added to the LB medium to induce expression of I-SecI endonuclease in pREDI; I-SecI endonuclease cut the I-SecI recognition site between the $\mathrm{Kan}^{\mathrm{r}}$ and $\mathrm{SacB}$ genes, which caused DNA breakage damage. The DNA repair system in the host identified the double-stranded DNA breakage, and then induced nearby homologous fragments to recombine for repair. The homologous recombination occurred between the $\mathrm{C}$ of $\mathrm{A}-\mathrm{C}-\mathrm{Kan}^{\mathrm{r}}-\mathrm{SacB}-\mathrm{B}$ and the gene downstream of UL18; thus, the full-length UL18 gene was deleted without any marker genes. Lastly, bacteria with successfully removed marker genes were selected by the SacB reverse maker gene coding levansucrase, which would result in bacterial death by catalyzing saccharose into fructose.

$B A C-H S V-1 \Delta U L 18$ failed to achieve viral rescue

We generated a UL18 knockout HSV-1 plasmid (BACHSV-1 $\Delta U L 18$ ) by two-step homologous recombination in vitro. Next, we transfected the BAC-HSV-1 $\triangle$ UL18 plasmid into Vero cells to obtain gene-deleted virus. The negative control (Vero+PBS) group showed no plaque formation or capsid protein VP5 expression. The positive control (BACHSV-1) group showed typical pathology (Fig. 3), with evident plaques (area enclosed by circular dotted box) and VP5 expression (area pointed by white arrow), verifying that the plaques were caused by virus infection. In contrast, the geneknockdown (BAC- $\triangle$ UL18) group expressed VP5 but no viral plaque formation was observed (Fig. 3). We conferred that the progeny mutant virus cannot assemble normally when UL18 is missing; thus, Vero cells did not form plaques. In order to further verify this mutant, we co-transfected BAC$\triangle \mathrm{UL} 18$ and pcDNA3.1-UL18 plasmids into Vero cells, which showed evident pathological reactions and VP5 expression (Fig. 3). This suggested that UL18 mutant HSV-1 is unable to package a regular capsid and is thus unable to produce a mature virion.

BAC-HSV-1 $\triangle U L 18$ plasmid vaccine enhances cellular immune responses in mice dramatically

We have constructed a novel attenuated HSV-1 DNA vaccine above, a deletion mutant viral vector, which required assessment of immune effects by animal trials before being applied clinically. Using the BAC-HSV-1 plasmid as a positive control and the BAC plasmid as a negative control to assess the immunogenicity of the BAC-HSV-1 $\Delta$ UL18 plasmid, the former produced infectious live virus in cells, unlike the latter.

Determination of serum specific antibody IgG (Table S1) and antibody neutralization assay (Table S2) were used to evaluate the humoral immune responses. Serum specific antibody IgG in vaccinated mice was determined by ELISA. The $N$ value of the negative groups of mice was $0.167>0.1$, and ratios $(P / N)$ of vaccinated groups and negative groups were $<2.1$, which showed that no significant difference between the two groups. Neutralization antibody assay indicated vaccinated groups of mice produced low antibody level, with a weak neutralization antibody ability. All in all, BAC-HSV-1 $\Delta$ UL18 plasmid vaccine was on a low level of humoral immune responses stimulation.

To test the vaccine DTH of BAC-HSV-1DUL18, we injected $10 \mu \mathrm{l}$ of inactivated HSV-1 suspension to the right subcutaneous auricle and $10 \mu \mathrm{l}$ supernatant of the frozen lysate to the left auricle for mice in each group following the final immunization and measured the thickness of auricles $48 \mathrm{~h}$ later. The thickness of auricles represented the activity of T-lymphocytes. Results showed that the thickness of auricles in BAC-HSV-1 $\Delta$ UL18-vaccinated mice had varied slightly but did not show any significant difference compared with the negative control (Fig. 4A). This indicated that the BAC-HSV-1 $\Delta$ UL18 vaccine was safe and caused no clear hypersensitivity in vivo.

Further experiments evaluated cell immune responses. Spleens were isolated and extracted, and the splenocytes were used for T-lymphocytes proliferation and cytokines detection. BAC-HSV-1 $\triangle$ UL18- and BAC-HSV-1-vaccinated 

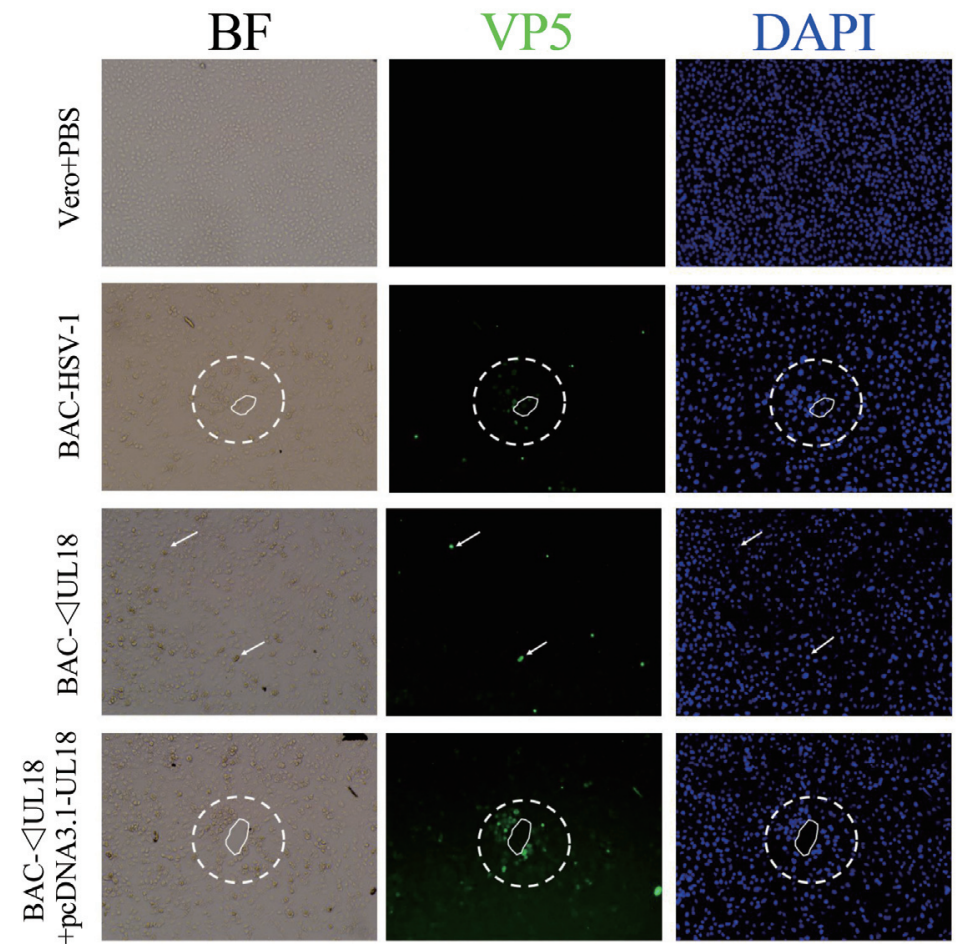

Fig. 3

Pathogenic detection of UL18 knockout mutant virus BAC- $\Delta$ UL18

Vero cells were seeded in 6 well culture plates and grown to $70 \%$ confluence for the different BAC plasmids as indicated in the figure transfection. After the positive control (BAC-HSV-1- transfected group) showed cytopathic effect, the cells were stained with VP5 antibody and DAPI. The area enclosed by the white circular dotted box indicates the plaque and the area indicated by white arrows shows VP5 expression. The fluorescence images were acquired using an OLYMPUS fluorescence microscope. BF: bright field.

(a)

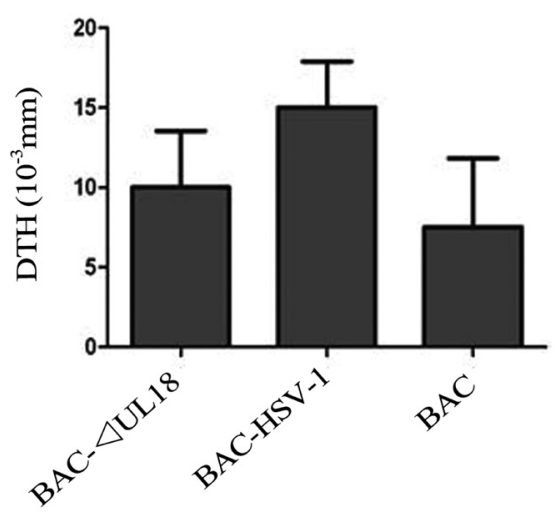

(b)

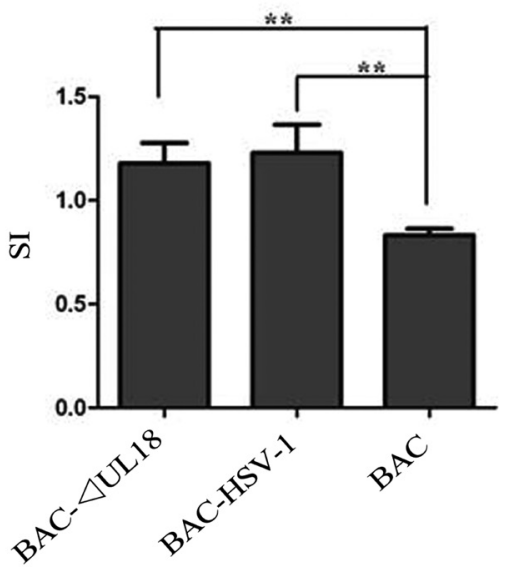

Fig. 4

Cellular immune responses in mice immunized with DNA vaccines

(a) The supernatants of plasmid BAC- $\triangle \mathrm{UL} 18$, BAC-HSV-1 and BAC, respectively, were injected into the right subcutaneous auricle, meanwhile inactivated HSV-1 suspension was injected into the left. The thickness of auricles was measured $48 \mathrm{~h}$ later using a vernier caliper. DTH is calculated by subtracting the measurement for the left subcutaneous auricle from the right one; (b) Proliferation of splenocytes from the immunized and control groups of mice was measured by the MTT cell proliferation assay. Data are represented as stimulation index (SI), which is calculated by dividing the absorbance (OD) observed in experimental group by OD observed in negative cells. 
groups showed specific T-lymphocyte proliferation responses, which significantly differed from those of the negative control (Fig. 4B). Cytokine detection was determined by ELISA. The results showed that IFN- $\gamma$ secretion levels of the BAC-HSV-1 $\triangle$ UL18 and BAC-HSV-1 vaccinated groups were significantly higher than those of the BAC group, while IL-2, IL-4, and IL-10 were not significantly different between BACHSV-1 $\triangle$ UL18 and BAC vaccinated groups (Supplementary Table 1). In summary, the BAC-HSV-1 $\Delta \mathrm{UL} 18$ vaccine has the potential to enhance the cell immune responses in vivo.

\section{Discussion}

Homologous recombination is one of the most common methods applied to gene knockdown. The bacterial markerless gene knockdown system constructed by our laboratory has many advantages compared to conventional virus knockdown methods: (i) it is very efficient and can accomplish gene knockdown within 3 weeks; (ii) the full-length target gene can be deleted without any other exogenous genes, avoiding the negative influences from exogenous marker genes; (iii) extracorporeal operation simplifies the flow; (iv) the process of gene knockdown is relative safe since there is no contact with live virus; and (v) the system could, in theory, be used in multiple gene knockdown or insertion. Thus, the bacterial markerless gene knockdown system is bound to have extensive application such as in the construction of multiple gene knockdown vaccines and the functional study of viral genes.

As of yet, no HSV vaccine has been effective in humans, although most such vaccines play a part in animal trials for stimulating inspiring protective immunity. What hinders the smooth development of HSV vaccines is the complexity of the HSV life cycle and immune mechanism and its latent character. Researchers studying HSV mostly conduct clinical evaluations of subunit, attenuated live virus, defective virus, and naked DNA vaccines. DNA vaccines, as the most novel vaccines, rely on a strategy to guide the recombinant eukaryotic expression vector into an organism and then stimulate protective immune responses by transcription and translation in the host (Pasette et al., 2003). DNA vaccines have many advantages compared with previous vaccines, including the impossibility of viral virulence resumption, persistent antigen expression, and low cost. In addition, the immune principle of DNA vaccines is similar to that of the natural viral infection.

$\mathrm{BAC}-\mathrm{HSV}-1 \Delta \mathrm{UL} 18$ is a type of DNA vector vaccine, where the $U L 18$ gene has been deleted through genetic engineering. Gene-deleted vaccines maintain the character of attenuated live virus vaccines, while preserving immunogenicity. The earliest mutant gene of HSV-1 was TK (Gordon et al., 1987), a primary HSV virulence gene, but its mutant attenuated vaccine has shown different immune protective efficacy in different species. The UL18 gene, as a component of the tegument, is an essential virulent gene of HSV-1, whose gene product VP23 stabilizes the structure of the tegument with VP5 and VP19C (Trus et al., 1996); thus, a UL18-deficient virus is not able to assemble into a live infectious virus. The virus rescue experiment showed that Vero cells did not grow progeny mutant virus when infected with the BAC-HSV$1 \Delta$ UL18 plasmid but were able to do so when infected with BAC-HSV-1. Additionally, BAC-HSV-1 $\Delta$ UL18 preserves other viral genes, including immunogenic components and glycoproteins.

The most important factor of HSV-1 in establishing lifelong infection is the character of immunologic escape. It is highly likely that DNA vaccines induce lifelong immunity and are involved in viral latency of the virus through cellular immunity, bearing both properties of prevention and therapy. Nevertheless, most non-replicating conventional vaccines cannot induce an effective cellular immune level. Lymphocyte proliferation is the most direct index of cellular immune level. The spleen, as the biggest immune organ, accounts for $25 \%$ of total lymphoid tissue. The cell proliferation assay verified that BAC-HSV-1 $\Delta$ UL18 could significantly stimulate lymphocyte proliferation and then induce an effective protective immune response in mice.

Cytokines detection indicates that BAC-HSV-1 $\Delta$ UL18 is able to improve the expression of IFN- $\gamma$ that induces the virus clearing response of the TH1 helper T cells. IFN- $\gamma$ and IL-4 represent a subset of TH1 and TH2 cytokines (Del Vecchio et al., 2007). IFN- $\gamma$, which has many important immune functions, significantly enhances the MHC-II expression of antigen presenting cells (APC) and strengthens the interaction of APC and T cells; therefore, it strengthens the production of antibodies and cytotoxic lymphocytes (CTL) (Ferris et al., 2006). In addition, IFN- $\gamma$ can suppress viral replication by inducing the infected cells to produce various anti-virus proteins. IFN- $\gamma$ is the activator of mononuclear macrophages, and strengthens the cytotoxicity of NK cells. Overall, IFN- $\gamma$ plays a crucial role in cellular immunity. However, BAC-HSV-1 $\Delta$ UL18 cannot stimulate expression of other cytokines including that of IL-2, IL-4 and IL-10. Improving the immunogenicity of BAC-HSV-1 $\Delta \mathrm{UL} 18$ requires the participation of other cytokines. At the same time, BACHSV-1 $\triangle$ UL18 does not appear to induce hypersensitivity, which may induce autoimmune diseases.

Furthermore, BAC-HSV-1 $\Delta$ UL18 cannot provide effective humoral immune responses in mice. The factors of determining the effectiveness of DNA vaccines depend on the choice of the target gene, the choice of vector and promoter, immunologic adjuvant, inoculation route, dose, and the organism itself. The development of the BAC-HSV-1 $\Delta$ UL18 vaccine requires further study to improve its humoral immune responses, like most other HSV-1 vaccines. Neverthe- 
less, there exists the potential significance of utilizing UL18 knockout in a HSV-1 DNA vaccine, which can be combined with the attenuated live virus vaccine by using the markerless gene knockout system in vitro.

\section{Conclusions}

In our study, combination of the pREDI plasmid and BACHSV-1 plasmid provides an effective bacterial markerless gene knockout system. Using the gene knockout system we successfully constructed a full-length UL18 gene knockout HSV-1 plasmid (BAC-HSV-1 $\Delta$ UL18) by two-step homologous recombination. BAC-HSV-1 $\Delta \mathrm{UL} 18$ induces great cellular immune effect in mice and indicates the potential of exploiting UL18 knockout HSV-1 as a DNA vaccine.

Acknowledgments. This work was supported by the National Natural Science Foundation of China (grant Nos. 81274170, 81573471); the International Cooperation Program of Guangdong Province of China (grant No. 2015A050502028); the Marine Strategic Emerging Industry Program (grant No. GD2013-B02-003); the Industry-University Collaborative Innovative Major Projects of Guangzhou (grant No. 201504291048224); the Collaborative Innovation Program of Guangdong Province of China (grant No. 2016A040402033) and the Biomedical Research And Development Center of Jinan University.

Supplementary information is available in the online version of the paper.

\section{References}

Boivin G, Goyette N, Sergerie Y, Keays S, Booth T (2006): Longitudinal evaluation of herpes simplex virus DNA load during episodes of herpes labialis. J. Clin. Virol. 37, 248-251. https://doi.org/10.1016/j.jcv.2006.09.006

Del Vecchio M, Bajetta E, Canova S, Lotze MT, Wesa A, Parmiani G, Anichini A (2007): Interleukin-12: biological properties and clinical application. Clin. Cancer Res. 13, 4677-4685. https://doi.org/10.1158/1078-0432.CCR-07-0776

Ferris RL, Whiteside TL, Ferrone S (2006): Immune escape associated with functional defects in antigen-processing machinery in head and neck cancer. Clin. Cancer Res. 12, 3890-3895. https://doi.org/10.1158/1078-0432. CCR-05-2750

Gierasch WW, Zimmerman DL, Ward SL, Vanheyningen TK, Romine JD, Leib DA (2006) Construction and characterization of bacterial artificial chromosomes contain- ing HSV-1 strains 17 and KOS. J. Virol. Methods 135, 197-206. https://doi.org/10.1016/j.jviromet.2006.03.014 Gordon YJ, Lance S, Ricardi T, Romanowski E, Araullo-Cruz T (1987): HSV-1 thymidine kinase negative vaccine: pathogenicity, protection, and perils. Curr. Eye Res. 6, 151-159. https://doi.org/10.3109/02713688709020083

Grunewald K, Cyrklaff M (2006): Structure of complex viruses and virus-infected cells by electron cryo tomography. Curr. Opin. Microbiol. 9, 437-442. https://doi.org/10.1016/j. mib.2006.06.016

Jensen K, Patel A, Larin A, Hoperia V, Saji M, Bauer A, Yim K, Hemming V, Vasko V (2010): Human herpes simplex viruses in benign and malignant thyroid tumours. J. Pathol. 221, 193. https://doi.org/10.1002/path.2701

Jin F, Li S, Zheng K, Zhuo C, Ma K, Chen M, Wang Q, Zhang P, Fan J, Ren Z, Wang Y (2014): Silencing herpes simplex virus type 1 capsid protein encoding genes by siRNA: a promising antiviral therapeutic approach. PloS One 9, e96623. https://doi.org/10.1371/journal.pone.0096623

Jin Q (2001): Medical Molecular Virology. Science Press, China, pp. 717-718.

Kennedy PGE, Chaudhuri A (2002): Herpes simplex encephalitis. Tidsskr. Nor. Laegeforen. 73, 237. https://doi.org/10.1136/ jnnp.73.3.237

Piacentini R, De Chiara G, Li Puma DD, Ripoli C, Marcocci ME, Garaci E, Palamara AT, Grassi C (2014): HSV-1 and Alzheimer's disease: more thanahypothesis. Front. Pharmacol. 5, 97. https://doi.org/10.3389/fphar.2014.00097

Pasetti MF, Barry EM, Losonsky G, Singh M, Medina-Moreno SM, Polo JM, Ulmer J, Robinson H, Sztein MB, Levine MM (2003): Attenuated Salmonella entericaserovar Typhi and Shigellaflexneri 2a strains mucosally deliver DNA vaccines encoding measles virus hemagglutinin, inducing specific immune responses and protection in cotton rats. J. Virol. 77, 5209-5217. https://doi.org/10.1128/ JVI.77.9.5209-5217.2003

Rodriguez F, Zhang J, Whitton JL (1997): DNA immunization: ubiquitination of a viral protein enhances cytotoxic T-lymphocyte induction and antiviral protection but abrogates antibody induction. J. Virol. 71, 8497-8503.

Trus BL, Booy FP, Newcomb WW, Brown JC, Homa FL, Thomsen DR, Steven AC (1996): The herpes simplex virus procapsid: structure, conformational changes upon maturation, and roles of the triplex proteins VP19c and VP23 in assembly. J. Mol. Biol. 263, 447-462. https://doi. org/10.1016/S0022-2836(96)80018-0

Weller S (2011): Alphaherpesviruses: Molecular Virology. Caister Academic Press 17.409.

Yu BJ, Kang KH, Lee JH, Sung BH, Kim MS, Kim SC (2008): Rapid and efficient construction of markerless deletions in the Escherichia coli genome. Nucleic Acids Res. 36, e84. https://doi.org/10.1093/nar/gkn359 


\title{
Supplementary information
}

\section{Construction, identification, and immunogenic assessments of an HSV-1 mutant vaccine with a $U L 18$ deletion}

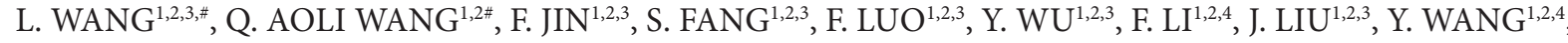 \\ J. JIN ${ }^{1,2}, \mathrm{X} . \mathrm{LIAO}^{1,2}, \mathrm{Z} . \mathrm{REN}^{1,2^{*}}, \mathrm{Y}$. WANG ${ }^{1,2^{*}}$
}

${ }^{1}$ Guangzhou Jinan Biomedicine Research and Development Center, National Engineering Research Center of Genetic Medicine, Jinan University, Guangzhou, Guangdong, P. R. China; ${ }^{2}$ Key Laboratory of Bioengineering Medicine, Guangzhou, Guangdong, P. R. China; ${ }^{3}$ College of LifeScience and Technology, Jinan University, Guangzhou, Guangdong, P. R. China; ${ }^{4}$ College of Pharmacy, Jinan University, Guangzhou, Guangdong, P. R. China

Received March 28, 2017; revised June 5, 2017; accepted April 11, 2018

Table S1. IgG of BAC- $\Delta$ UL18-, BAC-HSV-1- and BAC-vaccinated sera, respectively, and pre-immune sera as negative control were detected by ELISA

\begin{tabular}{|c|c|c|c|}
\hline Groups & $\begin{array}{c}\text { BAC-HSV-1 } \Delta \text { UL18 } \\
(\mathrm{P} / \mathrm{N} \pm \mathrm{SD})\end{array}$ & $\begin{array}{l}\text { BAC-HSV-1 } \\
(\mathrm{P} / \mathrm{N} \pm \mathrm{SD})\end{array}$ & $\begin{array}{c}\text { BAC } \\
(\mathrm{P} / \mathrm{N} \pm \mathrm{SD}) \\
\end{array}$ \\
\hline $1: 400$ & $1.218 \pm 0.032$ & $1.170 \pm 0.027$ & $1.135 \pm 0.016$ \\
\hline $1: 800$ & $1.178 \pm 0.029$ & $1.087 \pm 0.030$ & $0.893 \pm 0.017$ \\
\hline $1: 1600$ & $1.104 \pm 0.021$ & $1.363 \pm 0.119$ & $0.891 \pm 0.019$ \\
\hline $1: 3200$ & $1.209 \pm 0.043$ & $1.078 \pm 0.019$ & $0.905 \pm 0.014$ \\
\hline $1: 6400$ & $1.068 \pm 0.010$ & $1.183 \pm 0.014$ & $0.882 \pm 0.010$ \\
\hline 1:12800 & $1.023 \pm 0.007$ & $1.090 \pm 0.027$ & $0.926 \pm 0.015$ \\
\hline $1: 25600$ & $1.097 \pm 0.014$ & $1.064 \pm 0.023$ & $0.962 \pm 0.017$ \\
\hline
\end{tabular}

The antibody titers were determined as $\log$ of the reciprocal value of the highest serum dilution resulting in $\mathrm{OD}_{492}<0.1$ and $\mathrm{P} / \mathrm{N} \geq 2.1(P$ refers to the value of vaccinated mice OD; $N$ refers to the value of negative control mice OD).

Table S2. BAC- $\Delta$ UL18-, BAC-HSV-1- or BAC-vaccinated sera were mixed with HSV-1 virus (100 TCID50) by double dilution and then incubated with Vero cells

\begin{tabular}{|c|c|c|c|c|}
\hline $\begin{array}{ll}\text { Serum dilution } & \text { Groups } \\
\end{array}$ & $\begin{array}{c}\text { BAC-HSV-1 } \\
\quad \Delta \text { UL18 }\end{array}$ & BAC-HSV-1 & BAC & No-Treated \\
\hline 100 & ++++ & ++++ & ++++ & ++++ \\
\hline 200 & ++++ & ++++ & ++++ & ++++ \\
\hline 400 & ++++ & ++++ & ++++ & ++++ \\
\hline 800 & ++++ & ++++ & ++++ & ++++ \\
\hline 1600 & ++++ & ++++ & ++++ & ++++ \\
\hline 3200 & ++++ & ++++ & ++++ & ++++ \\
\hline 6400 & ++++ & ++++ & ++++ & ++++ \\
\hline HSV-1 control & ++++ & ++++ & ++++ & ++++ \\
\hline Cell control & - & - & - & - \\
\hline
\end{tabular}

Plus sign (+) represents positive cytopathic effect (CPE) and minus sign (-) represents negative of CPE. The infected Vero cells showed $75 \%-100 \%$ CPE, which was represented by “++++". 